Cinémas

Revue d'études cinématographiques

Journal of Film Studies

\title{
Une excentrique au coeur de l'industrie : Ray Lewis et le Canadian Moving Picture Digest
}

\section{Louis Pelletier et Paul S. Moore}

Volume 16, numéro 1, automne 2005

Femmes et cinéma muet : nouvelles problématiques, nouvelles méthodologies

URI : https://id.erudit.org/iderudit/013051ar

DOI : https://doi.org/10.7202/013051ar

Aller au sommaire du numéro

Éditeur(s)

Cinémas

ISSN

1181-6945 (imprimé)

1705-6500 (numérique)

Découvrir la revue

Citer cet article

Pelletier, L. \& Moore, P. S. (2005). Une excentrique au coeur de l'industrie : Ray Lewis et le Canadian Moving Picture Digest. Cinémas, 16(1), 59-90.

https://doi.org/10.7202/013051ar
Résumé de l'article

Ray Lewis fut, de 1918 à 1954, la rédactrice en chef du Canadian Moving Picture Digest, le premier journal corporatif relatif à l'industrie cinématographique canadienne. La personnalité et le parcours exceptionnels de Lewis peuvent expliquer sa présence - en apparence inusitée - à la tête d'une publication s'adressant à une industrie essentiellement masculine. Lewis milita ardemment dans les pages du Digest pour l'organisation et la défense des exploitants indépendants, de même que pour la création d'une culture nationale canadienne se démarquant du modèle américain. 


\title{
Une excentrique au cour de l'industrie: Ray Lewis et le Canadian Moving Picture Digest ${ }^{1}$
}

\section{Louis Pelletier et Paul S. Moore}

\begin{abstract}
RÉSUMÉ
Ray Lewis fut, de 1918 à 1954, la rédactrice en chef du Canadian Moving Picture Digest, le premier journal corporatif relatif à l'industrie cinématographique canadienne. La personnalité et le parcours exceptionnels de Lewis peuvent expliquer sa présence - en apparence inusitée - à la tête d'une publication s'adressant à une industrie essentiellement masculine. Lewis milita ardemment dans les pages du Digest pour l'organisation et la défense des exploitants indépendants, de même que pour la création d'une culture nationale canadienne se démarquant du modèle américain.
\end{abstract}

\section{ABSTRACT}

In 1918, Ray Lewis became editor of Canada's principal film trade journal, the Canadian Moving Picture Digest. She soon owned it, too, and stayed in charge until her death in 1954. If it was uncommon enough for a woman to be journalist and editor, it was truly exceptional for a woman to be in a powerful position in the film industry. Lewis' strong character carried into her work as self-appointed protector of Canadian exhibitors' independence. She used the Digest to agitate against Hollywood control, and tried to promote a distinctly Canadian culture of movie-going. 
L'un des documents les plus fréquemment cités par les historiens du cinéma au Canada est sans contredit le rapport déposé par Peter White le 30 avril 1931, au terme de la commission royale d'enquête visant à faire la lumière sur les pratiques monopolistiques de la Famous Players Canadian Corp. (alors la plus importante chaîne de salles de cinéma au Canada). La commission White faisait suite à plusieurs années de croisade en faveur des droits des exploitants indépendants et d'opposition aux activités monopolistiques de la Famous Players Canadian par le Canadian Moving Picture Digest, le premier journal corporatif de l'industrie cinématographique canadienne. Le nom de la propriétaire du Digest, Ray Lewis, qui fut également rédactrice en chef du journal de 1918 à 1954, est pourtant étrangement absent du rapport officiel de la commission ${ }^{2}$.

La participation de Lewis à cette enquête est cependant largement attestée par les comptes rendus des travaux de la commission, qui étaient régulièrement publiés dans la presse torontoise au cours de l'hiver 1931. Les journaux révèlent ainsi que Lewis avait été autorisée par le commissaire à procéder elle-même à certains interrogatoires et que, au cours des interrogatoires menés par White, elle ne se gênait pas pour interpeller les témoins du fond de la salle d'audience. L'un des plus importants témoins de la commission, le colonel Cooper de la Motion Picture Distributors and Exhibitors of Canada, en vint ainsi à qualifier Lewis "d'assistante-commissaire ${ }^{3}$ ». Par ailleurs, les comptes rendus non épurés des travaux de la commission White - dont on peut prendre connaissance en consultant la presse quotidienne de l'époque - ne font pas que rétablir la contribution de Lewis à l'enquête: ils décrivent également les circonstances ayant entraîné la disparition de la rédactrice en chef du Digest du rapport final.

Paradoxalement, cette disparition semble avoir été précipitée par le volume - tant en termes de quantité que de décibels des interventions de Lewis. L'exubérance de la rédactrice en chef du Digest est en effet rapidement utilisée contre elle par les dirigeants de la Famous Players Canadian, pour lesquels Lewis représente, tant par son statut d'initiée qu'en vertu de sa grande expérience de l'industrie, une bien plus grande menace que le 


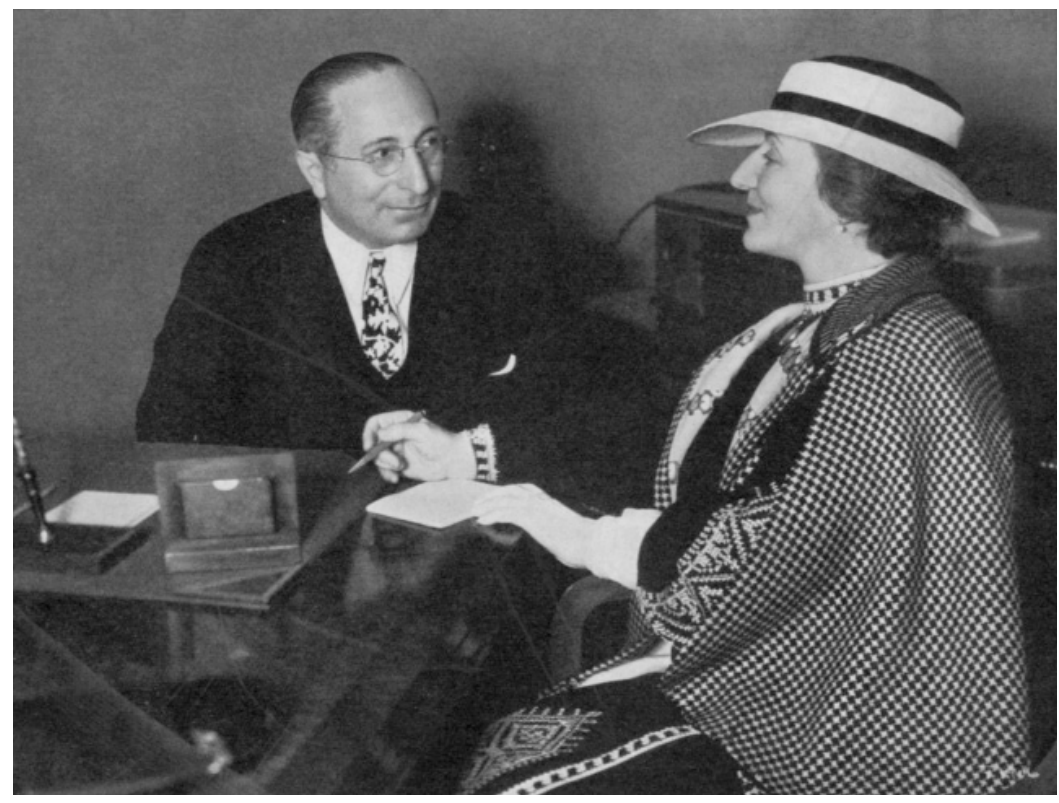

Hollywood, 1935: Louis B. Mayer dicte un message aux lecteurs du Digest (Canadian Moving Picture Digest, 25 mai 1935). (C) Collection Cinémathèque québécoise

commissaire White. Ce n'est cependant qu'après plusieurs jours d'interrogatoires serrés que le camp de la Famous Players Canadian trouve finalement la parade qui devait mettre Ray Lewis hors d'état de nuire. Alléguant que la légitimité de la commission se trouvait compromise par la participation de cette intervenante n'ayant pas de formation légale, l'un des membres du conseil d'administration de la Famous Players Canadian, I. W. Killam, cesse de collaborer à son interrogatoire par Lewis. Conscient de la valeur de Lewis pour la commission, White décide de s'accommoder de cette situation peu orthodoxe, et maintient le droit de parole de cette dernière. Il tente toutefois d'apaiser le camp de la Famous Players Canadian en décrétant que, dorénavant, les questions formulées par Lewis seraient relayées par lui aux divers témoins interrogés.

La situation ne manque cependant pas de dégénérer dès la reprise de l'interrogatoire de Killam, White et Lewis se querellant en effet aussitôt sur les questions admissibles dans le cadre des travaux de la commission. Le camp de la Famous Players 
Canadian, qui n'attendait que cela, saute alors sur l'occasion. Pendant que Killam, qui s'est retourné de façon à faire dos à Lewis, ricane, l'un des avocats représentant la Famous Players Canadian, saisissant sa veste, s'exclame: «Silly! Silly!», et claque la porte de la salle d'audience. Sur ce, Killam revient à la charge : il exige maintenant que les interventions de Lewis soient omises des transcriptions sténographiques des travaux de la commission. Pris entre deux feux, le commissaire White n'a alors d'autre choix que d'accéder à sa demande ${ }^{4}$. C'est ainsi que, bien qu'ayant été des plus audibles au cours des travaux de la commission, Ray Lewis en vint à perdre toute voix dans le rapport final.

Les adversaires de Ray Lewis seront nombreux au fil des ans à avoir recours à ce stratagème: plutôt que de répondre aux attaques et aux questions généralement bien informées de Lewis, on se contentera d'attirer l'attention sur l'exubérance et l'excentricité du personnage, sur son discours souvent intarissable et parfois décousu. Au même titre, le chercheur consultant pour la première fois le Digest à la recherche de données sur l'histoire du cinéma au Canada est en droit de se questionner sur la validité des opinions et des faits consignés dans les pages de la singulière publication de Lewis. Le présent article vise essentiellement à réinscrire la participation de Ray Lewis dans l'histoire du cinéma au Canada, ainsi qu'à souligner la valeur exceptionnelle du témoignage contenu dans les quelque deux mille numéros $\mathrm{du}$ Digest légués par Lewis. Nous tenterons d'abord, pour ce faire, de résumer brièvement le parcours de Lewis au cours des années qui précédèrent son arrivée au Canadian Moving Picture Digest, afin de démontrer que son accession à l'un des postes les plus en vue de l'industrie cinématographique canadienne ne fut pas le fruit du hasard. Nous examinerons ensuite le discours tenu par Lewis dans les pages du Digest, depuis le moment où elle prit en charge la publication, en 1918, jusqu’à la fin des années 1920 — une période correspondant à la fois à la dernière décennie du cinéma muet et au plus fort de la croisade de Lewis contre la monopolisation de l'industrie cinématographique. Nous tenterons finalement de situer Lewis dans le contexte social de son époque et, plus particulièrement, de voir comment elle put 
en venir à transgresser - et peut-être même à sciemment détourner à son avantage - certaines idées reçues concernant le rôle social de la femme.

\section{Ray Lewis : genèse d'un personnage}

"Messieurs, et Lady Ray Lewis": voici comment la veuve d'un vétéran de l'industrie cinématographique canadienne choisit d'ouvrir une allocution adressée aux convives d'un banquet tenu en 1952 par l'association Canadian Picture Pioneers ${ }^{5}$. Si, pendant la carrière de Ray Lewis, un certain nombre de femmes furent bel et bien actives dans le secteur de la production cinématographique - à titre d'actrices, bien sûr, mais aussi de monteuses, de scénaristes, de réalisatrices et de productrices ${ }^{6}$-, les postes clés des branches de l'industrie vouées à la distribution et à l'exploitation semblent avoir été exclusivement occupés par des hommes. Rappelons également qu'à cette époque, la presque totalité des films exploités au Canada étaient d'origine étrangère et que, par conséquent, les branches consacrées à la distribution et à l'exploitation représentaient presque à elles seules l'industrie cinématographique nationale. Dans ces circonstances, comment expliquer que le premier - et pendant plus d'une vingtaine d'années, le seul magazine corporatif relatif à l'industrie cinématographique canadienne ait été dirigé par une femme? Cette situation en apparence inusitée ne peut s'expliquer qu'à la lumière du parcours de Ray Lewis au cours des années ayant précédé son arrivée au Canadian Moving Picture Digest, en 1918.

Précisons d'emblée que la présente entreprise biographique (dont les résultats seront exposés plus en détail dans une publication à venir) n'échappe malheureusement pas aux problèmes de rareté et, surtout, de fiabilité des sources communs à la plupart des biographies traitant de pionniers du cinéma. La principale source d'information sur les trente-cinq premières années de la vie de Ray Lewis est un court texte autobiographique publié dans les pages du Digest en 1927. Intitulé "All about Myself ${ }^{7}$ ", ce document n'est guère plus qu'une courte suite d'anecdotes racontées sans réelle préoccupation d'ordre chronologique. Ce texte est également typique des mémoires de 
pionniers du cinéma en ce qu'il se montre tout aussi avare de dates et de faits vérifiables que prodigue d'anecdotes et de noms de personnalités prestigieuses - toutes prétendument côtoyées par notre héroïne au cours de son ascension vers les hautes sphères de l'industrie du divertissement. Très peu de documents d'époque pouvant venir corroborer, infirmer ou, simplement, enrichir la version de Lewis ont pu à ce jour être retracés.

Ray Lewis naît Rae Levinsky à Toronto en 1883. Elle est la seule fille d'une famille d'immigrants juifs fraîchement débarqués de Russie polonaise ${ }^{8}$. La demoiselle Levinsky est très tôt attirée par les feux de la rampe, au grand dam de sa famille de commerçants. La prospérité du commerce familial lui permet cependant de poursuivre son rêve d'une carrière sur les planches. Elle fait ainsi dès son plus jeune âge de nombreuses tournées, au cours desquelles elle côtoie plusieurs grands noms de l'industrie du spectacle. Après avoir interrompu pendant quelques années sa carrière théâtrale pour entreprendre, sous la contrainte parentale, des études universitaires ${ }^{9}$, Lewis effectue un retour sur scène vers 1907-1908. Elle acquiert alors une certaine notoriété grâce à un numéro de vaudeville intitulé Palmer \& Lewis in Footlight Flashes ${ }^{10}$, dans lequel elle enchaîne des démonstrations éclair de ses dons de comédienne, de chanteuse, de tragédienne et de danseuse classique. Lewis entame par ailleurs à la même époque une intense production de poèmes ${ }^{11}$ et de pièces de théâtre $^{12}$. En janvier 1915, elle produit l'une de ses pièces, The Other Woman, au prestigieux Massey Hall de Toronto, dans le cadre d'une soirée organisée par un groupe voué à la cause du suffrage féminin (figure 1). La critique remarque alors que cette pièce semble être, plus que toute autre chose, une tribune du haut de laquelle Lewis répand ses opinions sur une variété de sujets ${ }^{13}$. Au début de 1915, Lewis participe également à l'aventure de la Conness-Till, l'une des toutes premières compagnies à avoir tenté l'expérience de la production de films dans la région de Toronto. Lors de la cessation des activités de la Conness-Till au printemps de la même année ${ }^{14}$, Lewis s'établit à Flushing (N.Y.), où elle vend quelques histoires au studio Equitable, puis en Californie, où elle collabore avec le producteur Isadore Bernstein ${ }^{15}$ - un ami personnel - et la Fox 


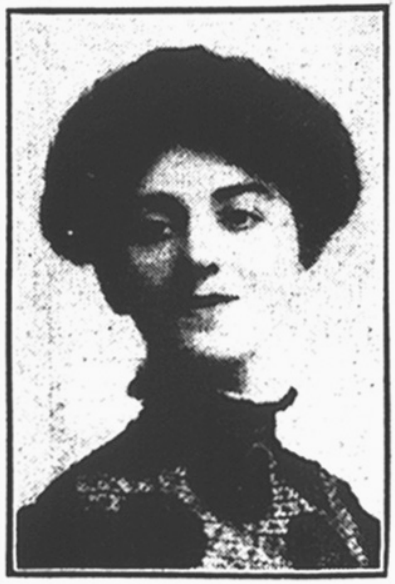

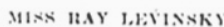

Figure 1. Ray Lewis en 1915 (The Globe [Toronto], 8 janvier 1915, p. 5).

Film ${ }^{16}$. Au moment où, en 1918, on lui offre la direction du Canadian Moving Picture Digest, Ray Lewis est âgée de trentecinq ans, n'est pas mariée et mène toujours une vie de bohème, entourée d'artistes et de gens de l'industrie du spectacle.

\section{Le Canadian Moving Picture Digest}

Dans les principaux pays producteurs de films, l'émergence d'une presse corporative relative à l'industrie cinématographique suit de très près l'apparition de salles consacrées à la présentation de vues animées sur une base régulière (les store fronts, nickelodeons et autres scopes), de même que celle des bureaux de distribution (les exchanges) les approvisionnant. Parmi les plus importants titres de cette presse, citons le Moving Picture World, publié à New York à partir de mars 1907, le Bioscope britannique, qui apparaît vers la fin de $1906^{17}$, et le Ciné-Journal français, publié à partir d'août $1908^{18}$. Le premier rôle de cette presse corporative est de conseiller les exploitants (une communauté comptant alors, rappelons-le, un grand nombre d'individus dépourvus de toute expérience de l'industrie du divertissement) sur une grande variété de sujets (pouvant aller de l'ajustement des tiges de carbone du projecteur aux meilleures façons de convaincre la clientèle féminine de se découvrir pendant le spectacle). La presse corporative agit également comme agent de liaison entre les différentes branches de l'industrie: 
tandis que les publicités, résumés et critiques qu'elle publie tiennent les exploitants informés des dernières productions offertes par les producteurs et distributeurs, les éditoriaux s'efforcent de coordonner les actions et objectifs de tous les membres de l'industrie; il importe pour le monde du cinéma de présenter un front uni à ses détracteurs. En somme, la presse corporative participe tant à la défense du cinéma qu'à la constitution d'une industrie juste et équitable envers chacun de ses membres.

Le cinéma ne manquait pas d'ennemis au Canada, et cela, dès 1908, année de la première campagne visant à forcer la fermeture des salles de vues animées le dimanche au Québec. Le premier journal corporatif canadien, le Canadian Moving Picture Digest, ne fut pourtant créé qu'en $1915^{19}$. Cette apparition tardive est de toute évidence attribuable au fait que l'industrie cinématographique canadienne se trouve dès l'origine absorbée par une industrie continentale contrôlée par des intérêts américains. Ce contrôle étranger ne représente pas une situation nouvelle: les différents circuits américains de salles de spectacles (destinées au théâtre, à la comédie burlesque ou au vaudeville), de cirques et de wild west shows, considéraient déjà depuis longtemps le Canada comme un marché domestique. Par conséquent, les journaux corporatifs de l'industrie américaine $\mathrm{du}$ spectacle (Variety, Billboard, Moving Picture World, Moving Picture News, Nickelodeon, etc.) publiaient tous des nouvelles concernant l'industrie cinématographique canadienne, et cela, dès l'ouverture des premiers nickelodeons. Cette presse américaine semble ainsi avoir, dans une large mesure, répondu aux besoins des exploitants de salles de cinéma canadiennes avant (et peut-être même après) l'arrivée du Canadian Moving Picture Digest. Rappelons qu'au milieu des années 1910, les films montrés au Canada sont presque tous importés - les productions américaines dominant le marché dès 1913-1914 — et distribués par les branches locales de diverses compagnies américaines. Plusieurs des salles canadiennes les plus prestigieuses sont de surcroît contrôlées par des circuits américains.

Le Canadian Moving Picture Digest est dirigé au cours de ses premières années de publication par Merrick R. Nutting. Bien 
que la publication soit basée à Montréal (le bureau du Digest se trouve dans l'édifice du théâtre Orpheum de la rue SainteCatherine Ouest), Nutting bénéficie de la collaboration de journalistes de Vancouver, Calgary, Toronto, Ottawa, Saint-Jean (N.-B.) et Halifax, qui agissent à titre de correspondants. La croissance du Digest n'en force pas moins Nutting à publier l'annonce suivante en janvier 1918:

If any of you fellows ever tried to get out a publication you know it is not a boy's job, especially if the detail of the whole thing has to be carried out by one person. The selling of advertising space, collecting and writing of advertising copy, the making of cuts and layouts, writing of editorial and news matter, as well as arranging the news matter from correspondents, is a man's work in itself. [...] The Digest has become too big for a one man proposition $[\ldots]^{20}$.

Des renforts arrivent quelques semaines plus tard, lors de l'acquisition par le Digest du Motion Picture Bulletin, que publiait la branche canadienne de la Universal Film Co. Le rédacteur en chef du bulletin Universal, Raymond S. Peck ${ }^{21}$, succède alors à Nutting à la tête du Digest ${ }^{22}$. Peu de temps après cette acquisition, le Digest suit le mouvement de centralisation amorcé par l'industrie cinématographique canadienne et transfere ses bureaux à Toronto. Peck n'occupera toutefois le poste de rédacteur en chef du Digest que pendant une brève période, puisqu'il quitte le magazine en septembre 1918 pour devenir gérant du bureau montréalais de la Mutual Films ${ }^{23}$. (Il dirigera plus tard, de 1920 à son décès, en 1927, le Canadian Moving Picture Bureau ${ }^{24}$.) À la suite de la démission de Peck, Nutting (qui, sous Peck, avait été le directeur de la publicité pour le Digest) se départit du Digest, qui devient alors la propriété de la Dominion Embossing and Printing Co. de Toronto. La nouvelle propriétaire du Digest se trouve alors forcée de trouver un autre rédacteur en chef dans les plus brefs délais. Le défi est de taille: comme l'écrivait Nutting, la direction du Digest n'est pas un «travail de garçon». Il n'en demeure pas moins que, de par sa grande expérience de l'écriture et de l'industrie du spectacle, Lady Ray Lewis représente 
pour les nouveaux propriétaires du Digest «l'homme de la situation ». Elle se retrouve donc à la barre du magazine à partir du numéro du 21 septembre $1918^{25}$. (Lewis deviendra également propriétaire du Digest dans les premiers mois de $1923^{26}$.)

Sous la direction de Lewis, le Digest se démarque rapidement des autres titres de la presse corporative. Dans l'un de ses premiers éditoriaux, la nouvelle rédactrice en chef revendique notamment le droit de consigner ses opinions dans les pages du Digest: "People that have red corpuscles in their veins are not Neutral. This is not the age of neutrality. [...] We do not wish to make our readers believe as we believe. What we are endeavouring to do is to stir them up to think for themselves ${ }^{27}$." Lewis n'hésite d'ailleurs pas à s'attaquer aux grandes questions de son époque dans les pages de son journal. Prétextant commenter une récente résurgence du mouvement de protestation contre l'exhibition ostentatoire du stars and stripes dans plusieurs films américains montrés à Toronto, elle se lance en février 1919 dans une charge griffithienne contre "l'intolérance", évoquant au passage les massacres des Arméniens et des Juifs polonais, les chrétiens jetés aux lions et la crucifixion de Jésus-Christ. Le mois suivant, dans un éditorial attaquant la propension des réformistes à établir un lien de causalité entre la popularité du cinéma et l'augmentation de la criminalité, Lewis n'hésite pas à y aller d'une hypothèse de son cru (les majuscules qui servent à marquer l'emphase constituent l'une des caractéristiques du style de la rédactrice du Digest) :

IF SOMEBODY IN POWER would think before they speak it might occur to SOMEBODY IN POWER that a state of War for four years might have something to do with an increase in crime.

When the protectors of women and children are taken away from them, when men are taken away from the spiritual influence of women, when these men for four years are trained to become efficient destroyers; and the women and children at home rejoice in THE BIGGEST KILLINGS, IT MIGHT HAVE A TENDENCY TO INCREASE CRIME ${ }^{28}$. 
Le contraste avec les éditoriaux de Walter R. Greene publiés quelques mois plus tard dans le Digest (qui dirigera la publication pendant le voyage d'étude effectué par Lewis en GrandeBretagne pour le compte de la chaîne Allen, entre avril 1919 et mai $1920{ }^{29}$ ) est plutôt frappant: l'un des éditoriaux de ce dernier porte sur "The Evil of Punch Holes" - sans aucun doute l'un des plus importants problèmes sociaux de l'aprèsguerre $^{30} \ldots$

L'une des stratégies les plus fréquemment employées par Lewis dans la défense de ses idées est le recours aux grands textes de la civilisation occidentale. Ses éditoriaux sont ainsi truffés de références à la mythologie grecque, à la Bible et aux œuvres de Shakespeare ${ }^{31}$. On peut notamment lire dans le numéro de Noël 1923 :

The Producer, the Distributor and the Exhibitor represent three wise men-the Public is the star which they must go forth to see [...]. All three united in purpose, going one way to find their Star of Fortune, will indeed find it, for it is said of "three" by Pythagoras, that it is the perfect number, the "beginning, middle, and end ${ }^{32}$."

Plusieurs références et métaphores employées par Lewis sont encore plus tarabiscotées. On peut notamment s'interroger sur la réception réservée par les petits commerçants constituant l'essentiel du lectorat du Digest à l'éditorial du 9 novembre 1918, intitulé "Mene, Mene, Tekel, Upharsin", dans lequel Lewis établit un parallèle entre les mésaventures du roi Balthazar de Babylone et la démesure des salaires touchés par les stars.

La tendance de Lewis à la logorrhée (tendance qu'elle assume pleinement ${ }^{33}$ ) n'arrange certes pas les choses. Plusieurs éditoriaux du Digest s'étirent sur deux, voire trois pages, et sont rédigés dans un style décousu laissant deviner l'imminence de l'heure de tombée. Comme nous l'avons précédemment signalé, ces vices de la rédactrice en chef du Digest permettront à plusieurs de ses adversaires, au cours de la commission White, de se défiler lorsque ses questions et critiques se feront trop pressantes. À cet égard, l'un des épisodes les plus instructifs - et les plus divertissants - de l'histoire du Digest est la guerre 
d'insultes opposant Ray Lewis à Sime Silverman — le tout aussi coloré rédacteur en chef du Variety —, guerre s'étant déroulée par éditoriaux interposés à la fin des années 1920. Lewis ouvre les hostilités en octobre 1927, en dénonçant vertement la publication par Variety, quelques semaines à peine après le décès de Marcus Loew, d'un numéro in memoriam lui étant dédié. Pour Lewis, cette entreprise illustre autant le mauvais goût que l'opportunisme et le manque d'éthique de Silverman ${ }^{34}$. Ce dernier répond à l'attaque de Lewis en publiant un article affirmant que, bien que l'industrie cinématographique canadienne ait connu une croissance rapide: "Exhibitors are without an organization and minus a trade paper of any merit ${ }^{35}$. " Ce à quoi Lewis réplique aussitôt dans les pages du Digest: "Attention, "Sime." We agree with you about the trade paper, it has no merit, because "merit" as you understand it is "money" [...]. We have often wondered, in reading your theatre grosses, if you would not be better "following the ponies" $[\ldots]^{36}$." Les réactions de Lewis à plusieurs autres articles et nouvelles publiés par Variety à la même époque seront encore plus directes. Le journal de Silverman est diversement attaqué par Lewis pour avoir ridiculisé la famille royale britannique, dénigré E. F. Albee ('un des héros de Lewis), ou encore révélé qu'une actrice de vaudeville avait eu un enfant en dehors des liens du mariage ( Kate Pullman Eugenic $\mathrm{Ma}^{37}$ »).

Silverman répond par une série de brefs commentaires condescendants ("The girl friend in Canada has been kinda laying off her idol, Albee. Wassa mat, sis; taken off the pay roll ${ }^{38}$ ?») qui contrastent avec les répliques de Lewis, s'étirant souvent sur plusieurs pages. Le rédacteur en chef de Variety ne manque d'ailleurs pas de se moquer du flot de paroles caractérisant les écrits de Lewis:

Have you ever seen a picture of your girl friend up in Canada? If she'd take that penholder away from her chin and stop trying to be journalistic [figure 2] she might have become smart enough to write in a gag pan and had it printed for nothing. [...] A 75-inch page would never hold what she's got on her mind ${ }^{39}$. 


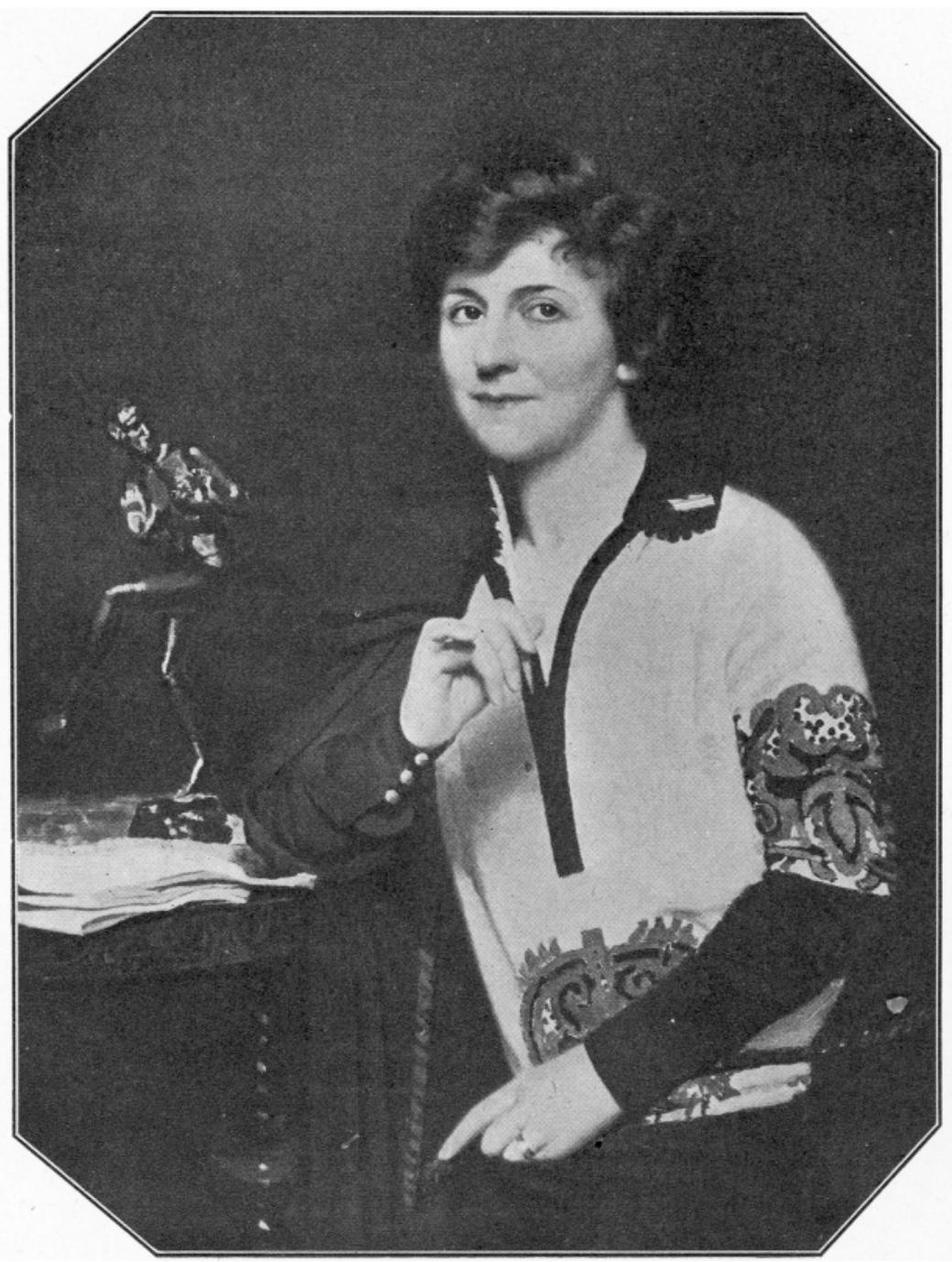

Figure 2. Portrait de Ray Lewis par son futur mari, Joshua Smith, reproduit sur la page frontispice du recueil de poèmes Songs of Earth (New York, Albert \& Charles Boni, 1917).

Silverman sera quant à lui l'objet de quelques caricatures issues de la plume de Lewis; la plus réussie est une reconstitution de l'un des passages les plus déchirants d'Uncle Tom's Cabin, et met en scène les deux rédacteurs incarnant les personnages principaux du roman (figure 3). 


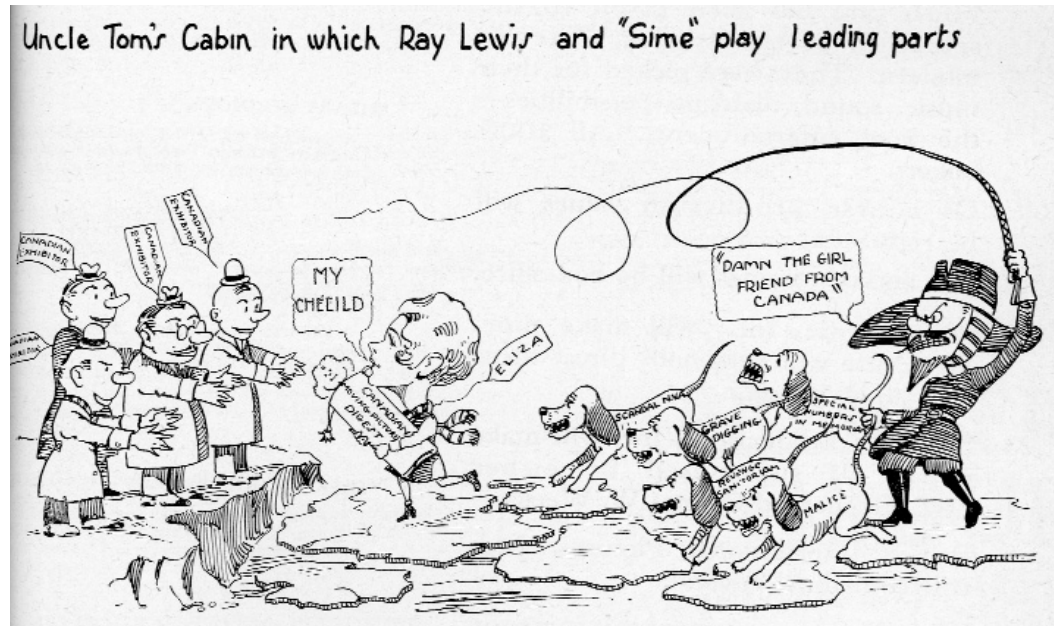

Eliza makes a getaway from Simon Legree who flourishes his ivhip while his bloodhounds howl - but all is in vain - for Canadian Exhibitors reach out their arms and ivelcome Eliza to freedom.

Figure 3. Canadian Moving Picture Digest, 21 décembre 1929. (C) Collection Cinémathèque québécoise

Si cet échange d'invectives entre Ray Lewis et Sime Silverman peut aujourd'hui sembler quelque peu futile, il en va évidemment autrement pour ce qui est de la croisade menée par Lewis dans les pages du Digest contre la monopolisation de l'industrie cinématographique canadienne. Le transfert du Digest à Toronto à la fin des années 1910 coïncidait en effet avec l'apparition de chaînes de salles canadiennes contrôlées à partir de la Ville reine et liées à des producteurs américains intégrés verticalement. La métaphore utilisée par Lewis pour illustrer l'influence parfois furtive de la plus importante de ces chaînes, la Famous Players Canadian Corp. de Nathan L. Nathanson, est celle de la pieuvre:

Monopolies are the antithesis of progress, initiative, individuality and independence. [...] If any one firm became so powerful in Moving Pictures that they were interested in every branch of it, tying up production, tying up exhibition, the raw product, the exchange [...], what chance would you, or any independent have?

This kind of beast is called an Octopus, its head is hidden in the crevice of a rock, in Picture Business the rock might be a subsidiary company, or a company 
operated by the Octopus under an assumed name, it waits from this vantage position for its prey, its tentacles forever reaching to seize, seize. [...] Fighting the tentacles, but reacts to the disadvantage of the victim, the head immediately increases the tension of the tentacle-hold. Go after the head ${ }^{40}$.

Lewis est très active tout au long des années 1920 dans le combat contre le monopole de la Famous Players Canadian ("The Combine»). Elle milite notamment pour l'organisation des indépendants, le Digest devenant en 1923 l'organe officiel de la branche canadienne de la Motion Picture Theatre Owners of America ${ }^{41}$. Lewis tente de stimuler la combativité des indépendants par de nombreux éditoriaux, n'hésitant pas au besoin à remettre en question leur virilité ("Are we men or are we Hermaphrodites, shrieking at opposition or competition like hysterical women ${ }^{42}$ ?") ou leurs capacités mentales ("You are supposed to be a scattered bunch of "Dumbells." Why have you earned such a reputation? There is only one answer, lack of organization $\left.[\ldots]^{43} . »\right)$

La position pro-indépendants du Digest lui attire rapidement les foudres de la Combine. Selon Lewis, relatant un entretien ayant vraisemblablement eu lieu vers juillet 1923 :

Mr. Nathanson told me if I did not behave he would put me out of business. He towered his six feet of anatomy over me, shook a fat forefinger in my face, and shouted that any advertiser using my paper would be cut off from play dates in his theatres. You see he lost his temper and gave his plans away; and I organized the M. P. T. O. of Canada $[\ldots]^{44}$.

Les menaces de Nathanson déclencheront une guerre ouverte entre le trust dirigé par la Famous Players Canadian et le Digest. Dépendants des salles contrôlées par Nathanson, la plupart des distributeurs n'ont d'autre choix que de retirer leurs publicités du Digest. Pour sa part, la First National Pictures, qui avait signé un contrat d'un an avec Lewis ${ }^{45}$, ne se contente pas d'interrompre ses livraisons de matériel publicitaire au Digest: elle force le magazine à aller sous presse avec une page couverture ne comportant qu'un simple point d'interrogation, et cela jusqu'à 
la fin de son contrat ${ }^{46}$. Faut-il se surprendre, dans ces conditions, de voir l'essentiel du contenu éditorial des numéros du Digest publiés au cours de l'année 1925 occupé par les attaques de Lewis contre Nathanson et le trust de la Famous Players Canadian? Une poursuite déposée en mars 1926 par Lewis ${ }^{47}$ se terminera cependant par une entente à l'amiable venant rétablir la paix entre le Digest et la Famous Players Canadian ${ }^{48}$. Lewis recevra même une lettre de félicitations d'Adolph Zukor pour son numéro de Noël $1926^{49}$.

Une autre cause défendue avec ferveur par Lewis dans les pages du Digest est celle de l'empire britannique. Cette préoccupation se manifeste dans plusieurs des reproches faits par Lewis au Variety de Silverman (qu'elle accuse d'avoir ridiculisé la famille royale ${ }^{50}$ ) et à la Famous Players Canadian (qui aurait refusé de faire bénéficier les productions britanniques de l'exploitation qu'elles méritaient [figure 4]). À cet égard, n'oublions pas que, dans un certain sens, le patriotisme représente la principale raison d'être du Canadian Moving Picture Digest ${ }^{51}$. Or, le patriotisme canadien-anglais de l'époque est toujours celui, pour reprendre les termes de Lewis, "of a British colony which became a British Nation ${ }^{52} »$. Le zèle patriotique de la rédactrice du Digest pourrait bien avoir été alimenté par son histoire personnelle - Ray Lewis Levinsky étant, rappelons-le, une immigrante juive de première génération. Après avoir connu la Russie tsariste, le Dominion du Canada avait dû représenter pour elle et sa famille une terre d'accueil des plus hospitalières. Qui plus est, Lewis épousa un citoyen britannique en 1920, le peintre portraitiste Joshua Smith, membre de la Royal Society of British Artists ${ }^{53}$.

La ferveur patriotique de Lewis n'exclut toutefois pas une certaine lucidité. Si Lewis fait ouvertement la promotion de la production cinématographique britannique ${ }^{54}$ (qu'elle a pu étudier entre 1919-1920 ainsi qu'en 1928, au cours de deux séjours outre-Atlantique), elle n'hésite cependant pas à en faire également la critique. Elle écrit notamment dans un éditorial de 1922 :

Although English productions have become much finer than they were some years ago, still the English-made picture, with some exceptions, is not as entertaining, 


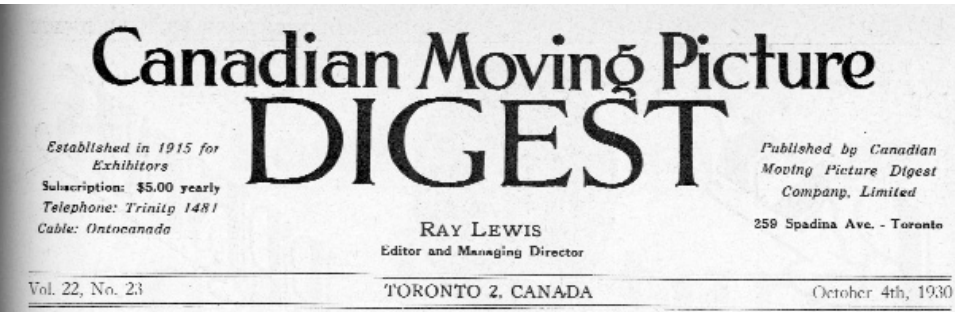

\section{"Come Out, Old Dear!"}
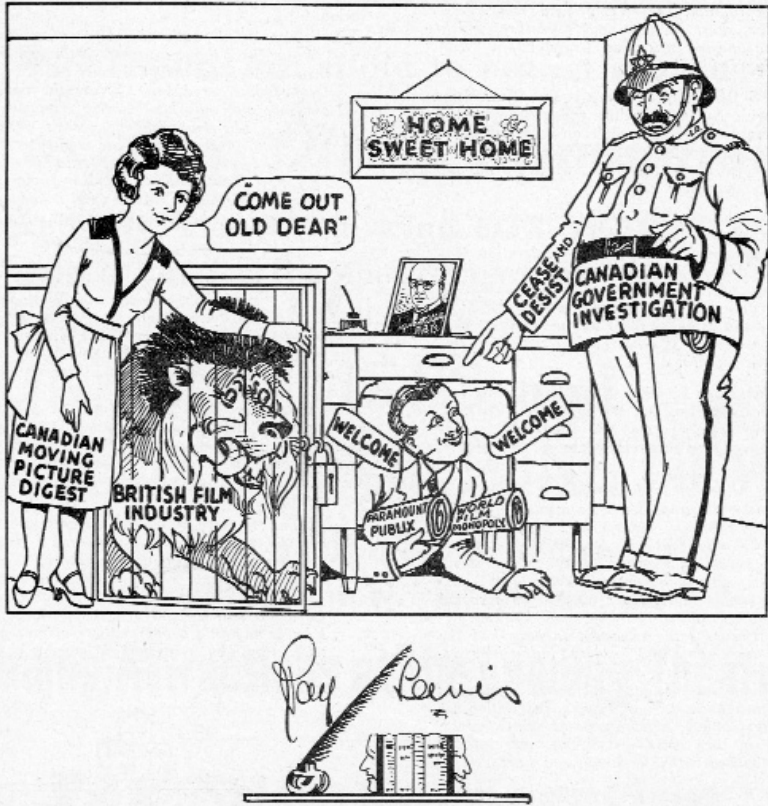

This is British Week in Toronto with Three British Films showing. Hurrah Investigation!

Figure 4. Canadian Moving Picture Digest, 4 octobre 1930. (C) Collection Cinémathèque québécoise

nor the quality which we find in pictures made on the American Continent. [...]

We had a press campaign in Canada, propaganda galore, and exhibitors' and exchanges' co-operation in regard to British Films, the public came, saw, but was not conquered ${ }^{55}$. 
Les velléités de productions cinématographiques canadiennes suscitent essentiellement les mêmes réactions chez Lewis. Si le Digest publie plusieurs éditoriaux affirmant la nécessité de favoriser la production nationale ( It is not healthy for a country to consume exclusively those products which are produced outside of its own domain $\left.{ }^{56} »\right)$, ou encore louant le travail de pionnier de Léo-Ernest Ouimet ${ }^{57}$, Lewis ne s'abstient toutefois pas de critiquer les activités des producteurs canadiens. Lorsque des allégations de malversations mettant en cause plusieurs individus impliqués dans la production de Carry on Sergeant! font surface au début de 1929, Lewis n’hésite pas à appeler un chat un chat: "The Digest's interpretation of graft "implies" converting of funds for uses not expressed in the financing, or obtaining of such funds ${ }^{58}$." Le patriotisme de Lewis est par ailleurs exempt de toute trace d'anti-américanisme, la rédactrice du Digest reconnaissant volontiers la qualité de la production américaine. Un poème de deux pages intitulé "Young Canada to Young America " ", publié dans le numéro spécial du Digest célébrant le cinquantième anniversaire de la confédération canadienne, illustre également la nature cordiale des sentiments entretenus par Lewis pour la nation américaine (de même que le style plutôt particulier du Digest...).

\section{Les poupées russes: l'actrice et la rédactrice}

La personnalité hors de l'ordinaire de Ray Lewis ne laissa pas ses contemporains indifférents. Comme l'écrit Hye Bossin dans la nécrologie de Lewis publiée dans le Canadian Film Weekly: "[...] she was a very unusual person and we will certainly not see her like again for many generations ${ }^{60} »$. Lewis ne se démarquait pourtant pas tellement par ses activités de rédactrice: au début du XX $\mathrm{XX}^{\mathrm{e}}$ siècle, le journalisme constituait en effet l'une des rares activités professionnelles ouvertes aux femmes, célibataires ou mariées. On relève ainsi dès 1912 plusieurs femmes journalistes chargées de la couverture de l'actualité cinématographique dans la presse quotidienne ${ }^{61}$. En 1914, la section "cinéma» d'un des quotidiens les plus influents d'Amérique du Nord, le Chicago Tribune, employait déjà deux femmes: Mae Tinee, chargée des potins et de la chronique «Mae Tinee's Answers to 
Movie Fans», et Kitty Kelly, chargée de la critique des films ${ }^{62}$. Au Canada, Alice Fairweather, du Standard de Saint-Jean (N.-B.), représentait pour sa part l'une des plus anciennes correspondantes du Digest ${ }^{63}$. Certaines de ces femmes journalistes devinrent de véritables célébrités (le Tribune publia notamment une bande dessinée ayant Kitty Kelly pour héroïne) et acquirent par conséquent une certaine influence. L'un des cas les plus connus et les mieux documentés est celui de Louella Parsons : après avoir été scénariste pour la compagnie Essanay de Chicago, Parsons fut embauchée en 1914 par le Chicago Herald $^{64}$, où elle publia d'abord une chronique intitulée "How to Write Photoplays». Ce n'est cependant qu'après être devenue, quelques années plus tard, l'échotière de Hollywood dans les journaux de William Randolph Hearst, que Parsons devint l'une des personnalités les plus influentes et les plus redoutées de l'industrie ${ }^{65}$.

L'apparition de ces femmes journalistes coïncide avec la transformation, décrite par Kathryn H. Fuller, de la figure archétypale du film fan. Celle-ci cesse en effet vers le milieu des années 1910 d'être celle du jeune homme entiché de technologie pour plutôt devenir celle de la jeune femme moderne passionnée pour les stars ${ }^{66}$. Cette mutation ne fut pas sans affecter les carrières de journalistes telles que Kitty Kelly et Louella Parsons qui, comme nous venons de le voir, durent graduellement adopter un rôle qui n'était initialement pas le leur: celui d'échotière. L'attribution d'une identité sexuelle féminine à l'archétype du film fan cadre ainsi parfaitement avec le phénomène plus global d'association des femmes à la culture de masse, décrit par Andreas Huyssen dans l'essai "Mass Culture as Woman: Modernism's Other »:

One aspect of the difference that is important to my argument about the gender inscriptions in the mass culture debate is that woman (Madame Bovary) is positioned as reader of inferior literature-subjective, emotional and passive-while man (Flaubert) emerges as writer of genuine, authentic literature-objective, ironic, and in control of his aesthetic means ${ }^{67}$. 
La féminisation du film fan constitue indéniablement une partie intégrante du processus d'institutionnalisation du cinéma. L'industrie cherche en effet, au tournant des années 1910, à rallier la classe moyenne à sa cause en courtisant la clientèle féminine, perçue comme étant la clé devant ouvrir ce vaste marché ${ }^{68}$. L'institution cinématographique se dote à cette fin de mécanismes permettant de contrôler tant les sujets traités à l'écran que la façon de construire et de gérer les salles de cinéma, espérant ainsi acquérir la respectabilité qui lui faisait cruellement défaut à l'époque du nickelodeon. Or, dans la société de l'époque, les mouvements de réforme - en faveur de la prohibition, du suffrage féminin, de l'amélioration des conditions de vie des classes ouvrières, etc. — sont pour la plupart associés à la sphère féminine. On remarque ainsi que le groupe de réformistes dénoncé par D. W. Griffith dans le "chapitre" moderne d'Intolerance (également connu sous le titre The Mother and the Law) est exclusivement composé de femmes d'un certain âge "The vestal virgins of Uplift", comme un des intertitres rédigés par Anita Loos les surnomme. Ray Lewis y va quant à elle de la sortie suivante dans un éditorial de 1919:

\begin{abstract}
WHEN MEN GET A MORAL STREAK IT IS SUSPICIOUS. A MORAL MAN, IN COMPARISON TO THE AVERAGE MORAL WOMAN, YOU KNOW AND EVERY MAN KNOWS THAT HE IS A RARE SPECIMEN, AND TO GET A CENSORSHIP BOARD OF MORAL MEN, HA, HA, EXCUSE US IF WE LAUGH ${ }^{69}$.
\end{abstract}

Ce n'est qu'après avoir nettoyé son image et gagné les faveurs de la nouvelle classe moyenne que le cinéma put en venir à représenter la quintessence de la culture populaire. Il est cependant important de noter que, tant cette conception du cinéma comme manifestation d'une culture populaire essentiellement féminine, que les croisades des groupes de réforme féminins ayant participé à l'institutionnalisation du nouveau média, demeurent en définitive asservies à la conception du cinéma comme entreprise commerciale. Or, ce champ d'activité appartenait plutôt à la sphère masculine dans la société du 
temps ${ }^{70}$. Ce cloisonnement n'était pas seulement symbolique: si on pouvait à cette époque retrouver un certain nombre de femmes journalistes, scénaristes ou réalisatrices, on n'en retrouvait cependant aucune à la tête de bureaux de distribution ou de chaînes de salles. Le caractère particulier de la situation de Ray Lewis découle précisément de cette situation : contrairement aux autres femmes journalistes écrivant sur le cinéma, Lewis s'adressait à un lectorat essentiellement masculin, puisque composé non pas d'artisans ou de fans de cinéma, mais bien de commerçants travaillant au sein de l'industrie cinématographique.

On s'en doute, l'irruption de Lewis dans ce «club» masculin ne fut pas sans susciter certaines réactions hostiles. La rédactrice en chef du Digest dut ainsi faire face au fil des ans à un certain nombre de railleries, de rumeurs et de commentaires condescendants. Bien que capable de ripostes cinglantes lorsqu'il s'agissait de défendre ses protégés, Lewis répliqua généralement avec détachement et humour à ces attaques personnelles. Au rédacteur en chef du Film Daily de New York, qui avait publié une fausse nouvelle affirmant qu'elle était sur le point de se rendre à Vienne afin d'y subir une "opération majeure ${ }^{71}$ ", Lewis réplique:

Vienna is $[\ldots]$ famous for making passe women uplift their chins, breasts and abdomens. Anything that has a tendency to fall, may fall in Vienna, but the experience is uplifting. [...]

In Vienna, a woman who still dreams of romance, even at sixty, can have "monkey-glands" grafted on her, where they will do the most good. Some call it "monkey-business," but I call it a major operation. I was never interested in majors, generals are more in my line, being ambitious, as for "monkey-glands," what would I do with them? It keeps me busy practicing self-control now. [...]

The article was headed "Toronto" indicating that the information came from there. Now I was born in Toronto, I know all their brands of humour, in fact very little humour is in Toronto, and what is there, has been monopolized by N. L. Nathanson, and myself, the rest of the people are Methodists. [...]

The humour, as presented in the Film Daily, was essentially New York humour ${ }^{72}$. 
Lewis suppose bien entendu l'existence d'une certaine connivence entre le Film Daily et le Variety de Silverman...

On comprend donc pourquoi celle qui était née Rae Levinsky préféra user dans les pages du Digest d'un nom - Ray Lewis masquant tant son identité sexuelle que son origine ethnique. Sur ce point, il est important de rappeler que, ayant d'abord été comédienne, Lewis avait pu très tôt dans sa carrière prendre connaissance des bénéfices pouvant être retirés de ces jeux de masques. Le numéro de vaudeville Footlight Flashes, au cours duquel elle alternait entre plusieurs formes de performances scéniques, constitue ainsi une parfaite métaphore de la carrière de Lewis. Cette dernière effectua au fil des ans de nombreux allers-retours entre ses multiples identités et ses différents rôles sociaux : l'artiste Ray Levinsky, la journaliste Ray Lewis, la politicienne ${ }^{73}$ et femme d'affaires Mrs. Joshua Smith. La journaliste Ray Lewis va même occasionnellement jusqu'à citer la politicienne Mrs. Joshua Smith dans les pages du Digest ${ }^{74}$. Cette mascarade semble avoir réellement servi Lewis en certaines occasions. Par exemple, Lewis raconte l'anecdote suivante dans les pages du Digest:

[Sam] Katz [of Paramount Publix] sent two of his henchmen to the Milwaukee Convention "to kick Ray Lewis in the pants," because of "The Spotlight's advice to exhibitors.

These henchmen happened to be talking to your editor and gave her this information, not connecting Miss Lewis with Ray Lewis.

When these Chicago "pant-kickers" found out that your editor did not wear pants, they hated to make a "bloomer" of the kicking; and gave her two ten dollar subscriptions to The Spotlight ${ }^{76}$.

Cela dit, Ray Lewis put en certaines autres occasions se prémunir contre les attaques de ses adversaires en mettant plutôt sa féminité à l'avant-plan. Contrairement aux autres publications de la presse corporative, qui tendaient à vouloir créer l'illusion d'une certaine "objectivité" journalistique (le Variety de Silverman représentant une possible exception), Ray Lewis n'hésitait pas à mettre à l'avant-plan son personnage et sa 
subjectivité dans les pages du Digest. La signature autographe de Lewis accompagne par exemple tous ses éditoriaux, tandis qu'une des principales rubriques du magazine, "Ray Presents», se trouve à chaque semaine ornée d'un des nombreux portraits de Lewis exécutés par son mari. Cette chronique permettait à la rédactrice du Digest de faire connaître à ses lecteurs des anecdotes et des opinions, mais aussi un certain nombre de bruits et de rumeurs concernant l'industrie. Des formules typiques de la presse féminine à potins ("The little birds that whisper stories to us ${ }^{77}$...") servent souvent d'introduction aux confidences et révélations obtenues par Lewis. En se présentant comme l'échotière de l'industrie cinématographique, Ray Lewis peut rendre publiques plusieurs développements importants que ses collègues masculins ayant des prétentions d'objectivité et d'impartialité n'auraient jamais pu annoncer ${ }^{78}$.

Le contraste entre la publication de Lewis et le Canadian Film Weekly publié à partir de 1936 par Hye Bossin (figure 5) est à cet égard des plus révélateurs. Alors que le Weekly — qui se veut une publication sobre et objective - évite de publier les informations confidentielles parvenant aux oreilles de ses collaborateurs, le Digest de Lewis se trouve à son meilleur au cours des périodes de bouleversements, lorsque bruits et rumeurs fusent de toutes parts ${ }^{79}$. Lorsqu'un conflit éclate à la tête de la Famous Players Canadian en 1929 et que Nathan L. Nathanson claque la porte de la compagnie, Lewis est là pour transmettre tous les bruits de coulisses aux distributeurs et exploitants canadiens - à qui elle ne manque par ailleurs pas d'expliquer les possibles conséquences de ce coup de théâtre sur leurs affaires. Une autre primeur relative aux activités de Nathan L. Nathanson obtenue par Lewis quelques années plus tard concerne le second départ de ce dernier de la Famous Players Canadian en 1941 (il avait repris la tête de la compagnie en 1933) et sa participation à la création de la chaîne Canadian Odeon Theatres. Le Digest ne manqua pas de rendre publics tous les détails de l'histoire, du mystérieux premier permis de construction délivré au nom de la nouvelle chaîne à l'affiliation de la dernière entreprise de Nathanson ${ }^{80}$. 


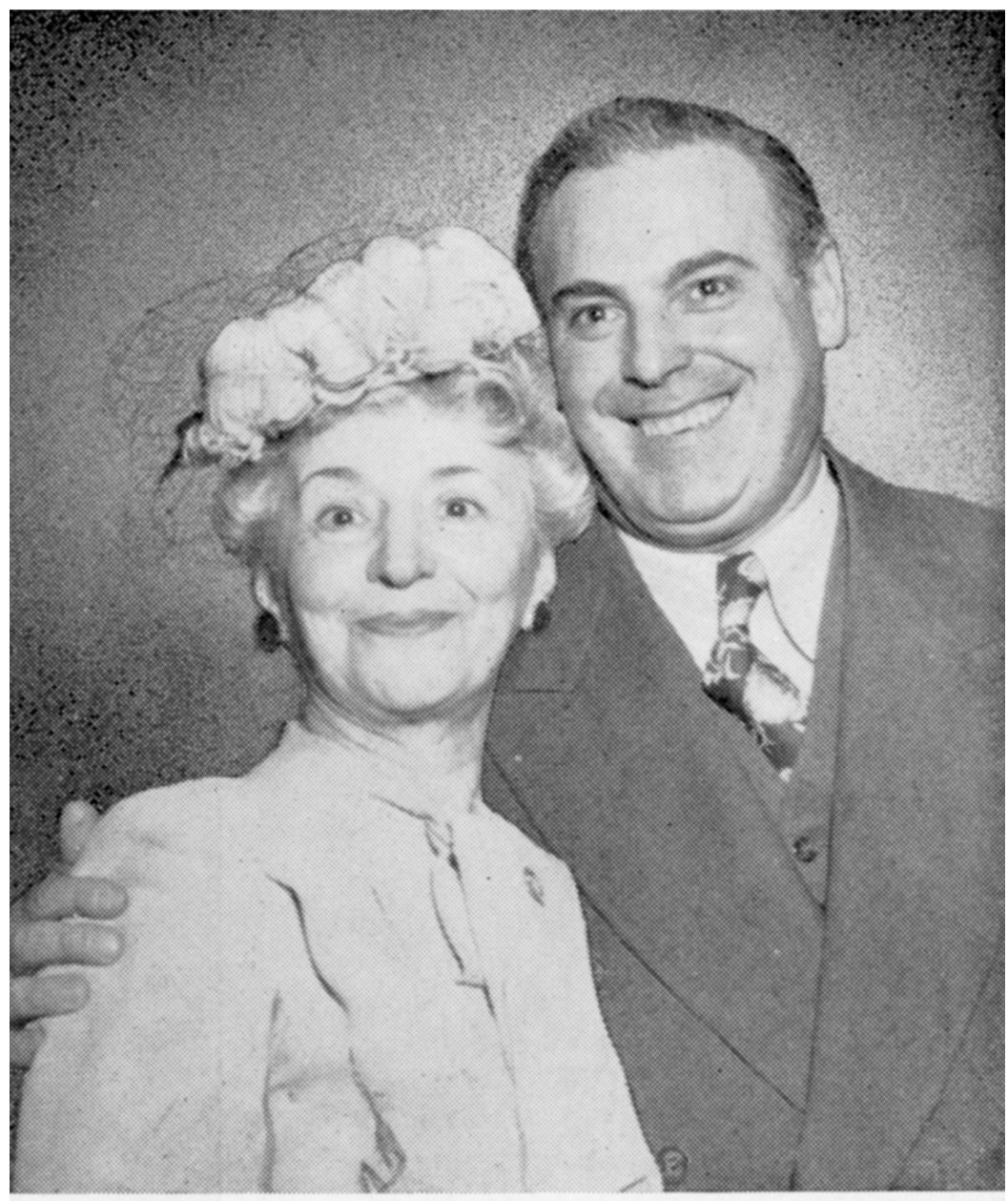

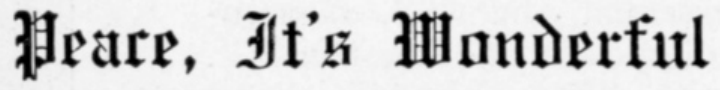 Congratulations, Ray HYE BOSSIN}

Figure 5. Ray Lewis et Hye Bossin, rédacteur en chef du Canadian Film Weekly (Canadian Moving Picture Digest, 13 avril 1946). (C) Collection Cinémathèque québécoise 


\section{Un héritage en question}

Comme l'a démontré le récit des événements survenus au cours des travaux de la commission White, l'exubérance de Ray Lewis put à l'occasion desservir le message qu'elle tentait de communiquer. Le caractère énergique et combatif de Lewis ne représente donc pas, en soi, un gage de l'influence du Canadian Moving Picture Digest. Les chiffres peuvent également être trompeurs à cet égard. En effet, bien que le Digest ait connu un tirage relativement important pour une publication canadienne spécialisée (1350 copies à la fin des années $\left.1920^{81}\right)$, on doit prendre en compte dans l'interprétation de cette donnée le fait que Lewis faisait parvenir son journal à l'ensemble des distributeurs et des exploitants canadiens, que ceux-ci aient manifesté leur intérêt en déboursant le prix d'un abonnement ou non (ce qui explique notamment pourquoi le Digest opérait toujours à perte plus de quinze ans après le début de sa publication). La promesse d'une pénétration totale du marché à ses annonceurs n'est pas la seule raison évoquée par Lewis pour justifier cette largesse ${ }^{82}$. En effet, si l'on en croit un éditorial de 1925, le profit ne représentait pas la principale motivation de la rédactrice du Digest:

I have quoted passages from the Bible, from mythology, classic and modern literature. I have been a high-brow and a low-brow, at your service. I have preached the principles of Independence until some exhibitors have been heard to say, "Is that from Shakespeare or the Bible?" I have sent you The Digest free, that is to a great number of you, some hundreds I should say, because I thought you needed me $[\ldots]^{83}$.

La cause embrassée par Lewis dépasse en fait largement celle des quelque milliers d'individus actifs au sein de l'industrie cinématographique canadienne: en participant à la création de cette industrie, Lewis entend apporter sa pierre à l'édification d'une identité nationale canadienne se démarquant des normes américaines $^{84}$. Lewis partage ainsi la vision d'un de ses contemporains et alliés, le pionnier montréalais Léo-Ernest Ouimet, qui avait notamment créé en 1919 le premier véritable journal d'actualités filmées canadiennes - le British Canadian Pathé 
News - pour des raisons manifestement plus patriotiques que commerciales. Lewis révèle d'ailleurs que :

[...] no one believed in The Digest more than Mr. Ouimet, no one gave The Digest greater support. [...] Mr. Ouimet's support consisted of this attitude, "Canada must have its own Motion Picture Trade Paper, published by Canadians;" and to this purpose Mr. Ouimet contributed twenty-five thousand dollars, five thousand dollars yearly for five years, giving The Digest a five year contract to indicate his faith in such an enterprise ${ }^{85}$.

Si le débat concernant l'importance de la contribution de Lewis à la création d'une industrie et d'une identité nationale canadienne demeure ouvert, il est un accomplissement de la rédactrice du Digest que l'on ne saurait remettre en question. En effet, en préparant un nouveau numéro du Digest à chaque semaine pendant près de quarante ans, Lewis nous a transmis une riche matière première n'attendant qu'à être mise au service d'une histoire restant largement à écrire: celle de l'industrie cinématographique canadienne.

\section{L'industrie honore une pionnière}

Ray Lewis semble avoir graduellement acquis le respect de l'industrie qu'elle prétendait servir. Cette reconnaissance pourrait notamment s'expliquer par le renouveau de ses activités professionnelles à la fin des années 1930. Lewis devint en effet une collègue de ses lecteurs le 26 octobre 1939, lors de l'inauguration du cinéma Pylon de Toronto (aujourd'hui connu sous le nom de Royal), dont elle était copropriétaire ${ }^{86}$. (Elle participera également quelques années plus tard à la direction d'un autre cinéma de Toronto, le Avenue.) De plus, Lewis se lança en 1940 dans la distribution de films en créant la Alliance Films. Opérant en marge des grandes compagnies hollywoodiennes (toujours intégrées verticalement au Canada), la Alliance Films se spécialisait dans l'importation de productions "en langues étrangères ${ }^{87}$ " et de films dits "d'exploitation ${ }^{88}$ ". Notons au passage que la compagnie de Lewis distribuait au Canada les productions de la Jack Broder Productions, qui 
comptait parmi ses réalisateurs le fils de celui qui avait été dans les années 1910 le gérant du studio Equitable, Felix Feist. Le propre fils de Lewis, James Lewis Smith, succédera pour sa part à sa mère à la tête de la Alliance Films, puis, après le décès de cette dernière, à la barre du Digest. Il maintiendra la publication en vie pendant quelques années avant de finalement la céder à Hye Bossin, qui procédera en 1957 à la fusion du Canadian Moving Picture Digest et du Canadian Film Weekly ${ }^{89}$.

Le dévouement de Ray Lewis pour l'industrie finit par susciter la reconnaissance du monde du cinéma, comme en témoignent certains gestes symboliques posés dans les dernières années de sa vie. Le 25 novembre 1953, l'association Canadian Picture Pioneers remet ainsi à la rédactrice du Digest une plaque reconnaissant sa contribution unique au développement de l'industrie cinématographique canadienne. À cette occasion, A. J. Mason, président du Motion Picture Industry Council of Canada, résume ainsi sa carrière:

\begin{abstract}
Ray Lewis-A name easily recognized as the most internationally known Canadian in the world of the film... She has kept the record of our joys and sorrows down through the years... Her life is so rich in intellectual adventure, and so full of the joy of living, that the rest of us must feel like dull drudges when we compare our lives with hers. Ray Lewis never asked for equal rights for women, instead she created them $[\ldots]^{90}$.
\end{abstract}

Lewis est hospitalisée quelques mois après cet hommage. De passage à Toronto, Adolph Zukor, qui avait pourtant affronté Lewis à plusieurs reprises à l'époque où il dirigeait Paramount Publix, se rend directement à son chevet après avoir été informé de sa maladie ${ }^{91}$. Ray Lewis décède finalement le 5 juillet 1954. De nombreux représentants de l'industrie cinématographique assisteront au service funèbre, dont Reuben W. Bolstad et J. J. Fitzgibbons de la Famous Players Canadian, David Griesdorf d'Odeon et Clare Appel de la Canadian Motion Picture Distributors Association ${ }^{92}$. Et, semble-t-il, ce n'était pas pour danser sur sa tombe.

Concordia University et University of Chicago 


\section{NOTES}

N.B. Abréviations utilisées dans les notes:

CMPD: Canadian Moving Picture Digest

MPW: Moving Picture World

Les citations tirées du Canadian Moving Picture Digest sont de Ray Lewis, à moins d'une indication contraire.

1. L'accès à certains documents ayant permis l'écriture de cet article nous a été facilité par Manon Viens, de la Médiathèque Guy-L.-Coté (Cinémathèque québécoise), et Ann Pelley, de la Mount Saint-Vincent University Library. Nous tenons à les remercier de leur précieuse collaboration.

2. Le témoignage de "Mrs. Joshua Smith» (le nom de femme mariée de Ray Lewis) occupe à peine la moitié de la page 154 du rapport (White 1931).

3. "Claims British Film Producer Reticent on Sales to Canada", Toronto Mail and Empire, 14 février 1931. Sur la participation de Ray Lewis aux travaux de la commission White, voir également "Exhibitors Not Forced to Overbuy", Toronto Telegram, 5 mars 1931.

4. «Killam Refuses to Make Replies to Mrs. Smith-Turns His Back and Answers Commissioner White, Who Relay Queries", Toronto Daily Star, 7 mars 1931; «Famous Players Voting Trust Status Unchanged by Trading Stock with Paramount Publix ", Mail and Empire (Toronto), 9 mars 1931.

5. La Canadian Picture Pioneers est un organisme de charité venant en aide aux vétérans de l'industrie tombés dans l'indigence. Lettre de Rae Allen à Nat Taylor, président de la Canadian Picture Pioneers, 5 novembre 1952 (York University Archives, fonds Nat Taylor 1999-036, boîte 005, dossier 51).

6. Là-dessus, voir Gaines 2002, p. 90.

7. CMPD, 13 août 1927 , p. 4, $6 ; 20$ août 1927 , p. 4, 10-11.

8. Recensement du Canada de 1901 : bobine T6497, district 116 (Toronto) A18.3.8, lignes 43 à 49. Les informations relatives aux résidences et aux professions sont tirées des annuaires de la ville de Toronto.

9. "Ray Lewis, Industry Leader, Passes», Canadian Film Weekly, 14 juillet 1954, p. 3; "Was Canada's First Lady of Theatre», Telegram (Toronto), 6 juillet 1954, p. 23.

10. Variety, 2 octobre 1909, p. 36.

11. Certains de ces poèmes ont été rassemblés dans The Cup of Civilization, from "Songs of the Universe" (Isadore Bernstein [dir.], Flushing, McConnell, 1915) et Songs of Earth (New York, Albert \& Charles Boni, 1917).

12. Pas moins de quatre pièces attribuées à Lewis ont été déposées à la Library of Congress de Washington au cours des seules années 1914 et 1915: In Shadow Land: A Fairy Play in Four Acts; The Other Woman: A Modern Comedy-Drama in Four Acts; All Men Are Liars: Satirical Drama in Four Acts et All Women Are Liars: One Act Drama. Elles peuvent être consultées sur la série de microfilms Canadian Dramas: Unpublished 1906-1942 (bobines 4 et 6), préparée par la Library of Congress de Washington.

13. News (Toronto), 4 janvier 1915, p. 10 (publicité); «Miss Ray Levinsky in Her Own Play", News (Toronto), 8 janvier 1915, p. 6; "The Other Woman", The Globe (Toronto), 8 janvier 1915, p. 6; "A Talented Author", The Globe (Toronto), 8 janvier 1915, p. 5.

14. La Conness-Till cessa ses activités en mai 1915 (Morris 1978, p. 51-54). Pour Lewis, cet échec n'a rien de bien mystérieux: "The King's Highway [sic] was out of focus and might have been the The King's Cabbage Garden, that is how close it was to being a good picture" ("All about Myself», CMPD, 20 août 1927, p. 10.) 
15. "Loyalty», $M P W, 10$ novembre 1917, p. 873.

16. "Enroute to California», CMPD, 23 février 1935; «Leon Trotsky AssassinatedWrote Story for Theda Bara», CMPD, 17 août 1940, p. 7.

17. Le premier numéro microfilmé par le British Film Institute porte le numéro 101 et est daté du 18 septembre 1908.

18. Sur les débuts de la presse corporative française, voir Toulet 1989.

19. Les numéros des deux premières années du CMPD semblent malheureusement perdus. Le premier numéro reproduit sur la série de microfilms préparée par la New York Public Library est celui du 24 novembre 1917. La Médiathèque Guy-L.-Coté de la Cinémathèque québécoise possède quant à elle une collection presque complète des numéros du CMPD des années 1920 et 1930.

20. "An Urgent Message to Canadian Exhibitors", CMPD, 5 janvier 1918, p. 6-7.

21. Dans "All about Myself», Ray Lewis affirme plutôt que le bulletin Universal était édité par un certain "Bill Bach" (CMPD, 20 août 1927, p. 10).

22. Merrick R. Nutting, "I Told You So!», CMPD, 19 janvier 1918, p. 6-7.

23. Voir Morris 1978, p. 159, citant le CMPD, 4 juin 1927, p. 14.

24. "Ray Peck Passes on over The Great Divide», CMPD, 4 juin 1927, p. 14. Voir Morris 1978, p. 159-165 (Morris affirme erronément que Peck fut le premier rédacteur en chef du $C M P D)$.

25. La page couverture de ce numéro (vol. $5, \mathrm{n}^{\circ} 12$ ) du CMPD est erronément datée du 21 août 1918. Notons par ailleurs que Ray Lewis affirma plus tard avoir été "silent editor" du CMPD sous Peck. Cependant, comme l'article dans lequel on trouve cette affirmation (CMPD, $1^{\text {er }}$ mai 1940) est truffé d'erreurs factuelles, et que l'on ne retrouve pas la "touche Lewis" dans les numéros du CMPD publiés avant septembre 1918, cette affirmation demeure très suspecte.

26. Le CMPD deviendra propriété américaine en mai 1926, lorsque Ray Lewis, subissant les effets du boycott orchestré par la Famous Players Canadian, devra céder son journal à la Exhibitors Review Publishing Corp. de George C. Williams. Elle reprendra cependant le contrôle du CMPD en novembre 1928 ("Give the Little Gal a Hand ", CMPD, 24 novembre 1928).

27. " $100 \%$ Canadian", CMPD, 2 novembre 1918.

28. "Silence Is Golden», CMPD, 29 mars 1919, p. 3.

29. Ray Lewis contribue au $C M P D$ tout au long de cette période. Elle reprendra son poste de rédactrice en chef en décembre 1920.

30. "Punch holes are intended to warn lazy or inattentive projectionists that the end of the reel is near" (Walter R. Greene, "The Evil of Punch Holes", CMPD, 2 octobre 1920, p. 15).

31. Voir le commentaire de Lewis dans "Subscriptions", CMPD, 12 septembre 1925.

32. "The Three Wise Men», CMPD, 22 décembre 1923.

33. On peut ainsi lire dans "All about Myself»: "I understand even up to the present, the disposition to talk with Ray, is one of her vices" (CMPD, 13 août 1927, p. 6).

34. "In Memoriam: An Open Letter to Variety», CMPD, 29 octobre 1927.

35. Variety, 4 janvier 1928, p. 24.

36. CMPD, 7 janvier 1928, p. 6.

37. Cité par Lewis dans "Another Example [of] Variety's Bad Taste», CMPD, 8 septembre 1928, p. 9. 
38. "Inside Stuff-Pictures", Variety, 15 mai 1929.

39. Variety, 26 juin 1929, p. 50.

40. "We're Agin It», CMPD, 5 août 1922. La pieuvre revient dans l'éditorial "The Trojan Horse" ( $1^{\text {er }}$ novembre 1924), ainsi que dans une caricature publiée le 13 octobre 1928.

41. "Montreal Dominion Headquarters for Moving Picture Theatre Owners of Canada", CMPD, 15 septembre 1923, p. 15.

42. "Muzzle the Lung-Exercisers», CMPD, 15 juin 1921, p. 2.

43. "The Montreal Convention", CMPD, $1^{\text {er }}$ septembre 1923.

44. "Harrison's Reports", CMPD, 25 avril 1925, p. 5. La date de l'entretien est présumée à partir de "Digest Sues for \$50,000 Damages" (CMPD, 6 mars 1926, p. 4).

45. "Louis Bache», CMPD, 12 juillet 1924.

46. Le boycott de la First National débute en décembre 1924 («Digest Sues for \$50,000 Damages », CMPD, 6 mars 1926, p. 4).

47. Ibid.

48. "The Searchlight», CMPD, 29 mai 1926, p. 2.

49. "Adolph Zukor Congratulates Digest Pointing out International Angle», CMPD, 15 janvier 1927, p. 16.

50. "Service from Variety", CMPD, 7 janvier 1928; "On Guard-Variety», CMPD, 14 janvier 1928.

51. À ce sujet, voir l'appel de Ray Lewis aux exploitants canadiens (CMPD, $1^{\text {er }}$ décembre 1921).

52. "The Building of a Nation", CMPD, 9 juillet 1927.

53. "Marriage of Miss Lewis", CMPD, $1^{\text {er }}$ novembre 1920, p. 12. Une photographie montrant Joshua Smith au travail est reproduite dans le CMPD du 25 mai 1935. Si l'on en croit son témoignage, Lewis profita de son voyage de noces pour écrire un scénario en collaboration avec le célèbre explorateur (et fabulateur) britannique F. A. Mitchell-Hedges ("All about Myself», CMPD, 20 août 1927, p. 11).

54. Voir par exemple la série d'articles «Educating the Public for British Pictures», publiés par Lewis dans le Digest à partir du 3 avril 1920.

55. "They Came-They Saw-But Were Not Conquered", CMPD, 30 septembre 1922.

56. "Money of Glory", CMPD, $1^{\text {er }}$ mars 1922.

57. Ibid.

58. "The Digest Was Right", CMPD, 23 février 1929. Voir aussi "Carry On Sergeant ", CMPD, 12 janvier 1929.

59. "Young Canada to Young America», CMPD, 9 juillet 1927, p. 50-51.

60. "Ray Lewis, Industry Leader, Passes», Canadian Film Weekly, 14 juillet 1954, p. 3.

61. Le plus ancien cas rapporté est celui de Gertrude M. Price, «Moving Picture Expert» du Des Moines News (Iowa). Voir à ce sujet Abel 2001 (p. 59).

62. Le premier article de Kitty Kelly fut publié par le Chicago Tribune le 3 juillet 1914.

63. Le rapport de la commission White contient d'autres détails sur la carrière d'Alice Fairweather (White 1931, p. 211).

64. La publication de la chronique de Parsons dans le Chicago Herald débuta le 13 décembre 1914. 
65. Sur la carrière de Parsons, voir Barbas 2005.

66. Voir Fuller 1996 (p. 145-149).

67. Voir Huyssen 1986 (p. 44-62).

68. Là-dessus, voir Hansen 1991 (p. 121-125) et Pelletier et Russell 2004.

69. "Fools Rush in ", CMPD, 15 mars 1919.

70. Voir Huyssen 1986 (p. 45).

71. "Ray Lewis Going Abroad", Film Daily, 13 juin 1928, p. 2.

72. CMPD, 23 juin 1928, p. 4. Voir également "It Is a Good Thing Cows Don't Fly", CMPD, 22 mai 1926.

73. "Mrs. Joshua Smith" se présente en 1930 à l'investiture du Parti conservateur dans la circonscription du Centre-Ouest de Toronto (CMPD, 12 juillet 1930, p. 14).

74. Voir par exemple "Have Women a Political Conscience?», CMPD, 12 juillet 1930 , p. 14.

75. Publié par Ray Lewis pendant son association avec la Motion Picture Theatre Owners of America au milieu des années 1920, The Spotlight était la contrepartie américaine du Canadian Moving Picture Digest.

76. $C M P D, 7$ juin 1930 , p. 4.

77. "The Searchlight», CMPD, 13 mars 1926.

78. Un bâton de fou du roi jouxte d'ailleurs chacun des éditoriaux du CMPD publié à partir de mai 1929.

79. Les styles différents de Lewis et de Bossin purent cependant être façonnés tant par les standards journalistiques de leur époque respective que par certains stéréotypes relatifs aux rôles sexuels. Schudson (1978) remarque ainsi que, dans le sillage de la Première Guerre mondiale, le journalisme vint à être ouvertement considéré comme un outil de propagande. Ce n'est qu'au cours des années 1930 que l'objectivité en serait venue à être reconnue comme la première valeur journalistique.

80. "Two New Theatres Announced for Kingston", CMPD, 25 janvier 1941; "Odeon Theaters of Canada Will Operate Dominion-Wide Chain", CMPD, 22 février 1941; "Lansdowne, Toronto, to FPC-Odeon takes Capitol Brampton", 21 juin 1941; "Pylon and Avenue Join with Famous Players Canadian", CMPD, 14 juin 1941. Sur ce sujet, voir Moore 2003.

81. CMPD, 11 janvier 1930, p. 6 ; CMPD, 24 mars 1928, p. 6.

82. «Good Times!», CMPD, 9 septembre 1922.

83. "Subscriptions", CMPD, 12 septembre 1925.

84. Là-dessus, voir l'appel publié par Lewis dans le CMPD du $1^{\text {er }}$ décembre 1921.

85. CMPD, 5 novembre 1927, p. 6.

86. "A Dream Come True», CMPD, 28 octobre 1939, p. 1 ; «Open Pylon Theatre, Toronto", ibid., p. 5.

87. Le Pylon était situé dans un quartier de Toronto comptant une large population d'origine italienne. On peut donc présumer que la Alliance fut créée afin de procéder à l'importation de films répondant aux besoins particuliers de cette clientèle.

88. Film Weekly 1952-1953 Year Book of the Canadian Motion Picture Industry, Hye Bossin (dir.), p. 115.

89. "The Digest Will Stop Publication", Canadian Film Weekly, 27 février 1957, p. $1,3$.

90. Film Weekly 1954-1955 Year Book of the Canadian Motion Picture Industry, Hye Bossin (dir.), p. 29.

91. "Ray Lewis, Industry Leader, Passes», Canadian Film Weekly, 14 juillet 1954, p. 3. 
92. "Ray Lewis Being Widely Mourned», Canadian Film Weekly, 21 juillet 1954, p. 3.

\section{RÉFÉRENCES BIBLIOGRAPHIQUES}

Abel 2001: Richard Abel, "A Marriage of Ephemeral Discourses: Newspapers and Moving Pictures", Cinema \& Cie., nº 1, 2001, p. 59-83.

Barbas 2005: Samantha Barbas, The First Lady of Hollywood: A Biography of Louella Parsons, Berkeley/Los Angeles/London, University of California Press, 2005.

Famous Players: Dossier de presse de la Famous Players, Médiathèque Guy-L.-Coté, Cinémathèque québécoise.

Fuller 1996: Kathryn H. Fuller, At the Picture Show: Small-Town Audiences and the Creation of Movie Fan Culture, Washington/London, Smithsonian Institution Press, 1996.

Gaines 2002 : Jane M. Gaines, "Of Cabbage and Authors", dans Jennifer M. Bean et Diane Negra (dir.), A Feminist Reader in Early Cinema, Durham/London, Duke University Press, 2002, p. 88-118.

Hansen 1991 : Miriam Hansen, Babel and Babylon: Spectatorship in American Silent Film, Cambridge/London, Harvard University Press, 1991.

Huyssen 1986: Andreas Huyssen, After the Great Divide: Modernism, Mass Culture, Postmodernism, Bloomington/Indianapolis, Indiana University Press, 1986.

King Hanson 1988: Patricia King Hanson (dir.), The American Film Institute Catalog of Motion Pictures Produced in the United States: Feature Films, 1911-1920, Berkeley/Los Angeles/London, University of California Press, 1988.

Moore 2003: Paul S. Moore, «Nathan L. Nathanson Introduces Canadian Odeon: Producing National Competition in Film Exhibition", Canadian Journal of Film Studies, vol. 12, n' 2, 2003, p. 22-45.

Morris 1978 : Peter Morris, Embattled Shadows: A History of Canadian Cinema, 1895-1939, Montréal, McGill/Queen’s University Press, 1978.

Pelletier et Russell 2004 : Louis Pelletier et Catherine Russell, «Ladies Please Remove Your Hats': Fashion, Moving Pictures and Gender Politics of the Public Sphere, 1907-1911 ", Living Pictures, n 3, 2004, p. 61-84.

Schudson 1978 : Michael Schudson, Discovering the News: A Social History of American Newspapers, New York, Basic Books, 1978.

Sperdakos 1999: Paula Sperdakos, "Canada's Daughters, America's Sweethearts: The Careers of Canadian "Footlight Favorites" in the United States", Theatre Research in Canada/Recherches théâtrales du Canada, vol. 20, n 2, 1999.

Toulet 1989: Emmanuelle Toulet, "Aux sources de l'histoire du cinéma... Naissance d'une presse sous influence", dans Restaurations et tirages de la Cinémathèque française $I V$, Paris, Cinémathèque française, 1989, p. 14-25.

White 1931 : Peter White, Combines Investigation Act: Investigation into an Alleged Combine in the Motion Picture Industry in Canada, Report of Commissionner, April 30, 1931, Ottawa, F. A. Acland, Printer to the King, 1931. 